\title{
TESTES DE LIMIARES PARA A SEGMENTAÇÃO DE IMAGENS SPOT-5 VISANDO A DETECÇÃO DE PLANTIOS FLORESTAIS
}

\author{
Image segmentation of SPOT-5 satellite tests applied to manmade forest
}

\author{
Ana Paula Dalla Corte ${ }^{a}$, Fernando Luís Dlugosz $^{\mathrm{b}}$, Jorge Antônio Silva Centeno $^{\mathrm{c}}$ \\ a Engenheira Florestal, M.Sc., Doutoranda da Universidade Federal do Paraná. Bolsista CNPq. Curitiba, PR - Brasil, e-mail: \\ anacorte@ufpr.br

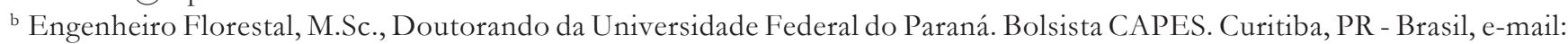 \\ fernando@floresta.ufpr.br \\ c Engenheiro Civil, Dr., Professor do Departamento de Geomática da Universidade Federal do Paraná. Curitiba, PR - Brasil, e- \\ mail:coceano@terra.com.br
}

\begin{abstract}
Resumo
A segmentação de imagens de alta resolução é uma técnica bastante usada nos dias de hoje. Nesta técnica os pixels das imagens podem ser avaliados em função da descontinuidade e similaridade dos tons de cinza. Na definição dos limiares para os critérios utilizados pelo algoritmo é necessário que se conheça as características do alvo avaliado, visando, desta forma, obter valores que melhor os identifiquem. Assim, o objetivo deste trabalho foi testar os melhores limiares para imagens SPOT- 5 com resolução espacial de 5 metros, aplicadas ao reconhecimento de plantios florestais. Os testes foram realizados com o software SPRING, utilizando-se o algoritmo de crescimento de regiões. Foi executada uma interpretação visual como referência na avaliação dos resultados. A forma dos segmentos foi usada para auxiliar na escolha dos melhores limiares. O par de limiar mais próximo do referencial foi o 45 de similaridade com 300 de área. Pode-se afirmar que a área, o perímetro e o coeficiente de compacidade contribuíram para a escolha dos pares de limiares, porém, não conseguiram expressar claramente o melhor par.
\end{abstract}

Palavras-chave: Sensoriamento remoto; Cultivo florestal; Análise visual.

\section{Abstract}

The high resolution images segmentation is a technique commonly used nowadays. In this technique the image pixels can be appraised as function of the gray levels and also in similarity indexes. In the threshold definition of the utilized criteria by the algorithm is necessary to know each appraised object characteristic, aiming to obtain values that better identify them. Thus, the 
objective of this work was to test the best threshold for SPOT-5 images, with 5 meters spatial resolution, applied to the recognition of manmade forest. The tests were accomplished with the software SPRING, using the region growth algorithm. A visual interpretation was made as parameter in the results evaluation. The segments shape was used to aid in the choice of the best thresholds. The threshold pair that was nearest to the referential was the 45 of similarity with 300 of area. It can be affirmed that the area, the perimeter and the compactness coefficient contributed for the thresholds pairs choice, perhaps, they could not express clearly the best pair.

Keywords: Remote sensing; Manmade forest; Visual analysis.

\section{INTRODUÇÃO}

Com o surgimento de satélites de melhor resolução espacial, há a necessidade de aprimorar as técnicas utilizadas no tratamento de dados oriundos dos sensores ora existentes, de maneira a permitir investigar mais detalhadamente o conteúdo de informações contidas nas imagens. Para isto, o constante desenvolvimento de técnicas computacionais vem tentando cada vez mais simular a capacidade do sistema visual humano em reconhecer padrões, baseando-se em propriedades espectrais e espaciais dos dados remotamente sensoriados. Dentre as vantagens do uso do computador no reconhecimento dos padrões, em relação à interpretação visual, uma reside na capacidade que estes têm de processar grande volume de informações presentes na imagem, tarefa esta limitada ao ser humano (MOREIRA, 2001).

Uma técnica bastante difundida no meio acadêmico nas últimas décadas, diz respeito à segmentação de imagens de alta resolução, ao invés de se envolver apenas nos aspectos de delimitação de áreas e caracterização do uso do solo.

A segmentação automática, em geral, baseia-se nas características de descontinuidade e similaridade dos tons de cinza de uma imagem. O método que avalia a descontinuidade considera a mudança abrupta dos valores de cinza e o método por similaridade fundamenta-se na agregação de pixels em função da sua semelhança com os pixels vizinhos (GONZÁLES; WINTZ, 1987). A segmentação por crescimento de regiões se baseia neste segundo método.

Conforme Venturieri e Santos (1998), a operação de segmentação por crescimento de regiões consiste em agrupar pixels com características similares em termos tonais e texturais, formando regiões homogêneas. Desta forma, a imagem é particionada em um conjunto de regiões correspondentes às feições da superfície terrestre, que irão servir de base para a análise e cartografia temática. Os resultados desse processo são áreas com aspecto contínuo, onde cada área possui características espectrais bem diferentes das vizinhas que a cercam.

De acordo com Coutinho (1997), a definição de valores para os limiares na segmentação de imagens depende, principalmente, do conhecimento dos padrões de repartição espacial dos objetos de cada área e da definição da generalização cartográfica ideal, em função da escala cartográfica da abordagem realizada. Contempla uma etapa que exige maior atenção devido à inexistência de valores padronizados para as variáveis do algoritmo e, também, pode influenciar diretamente na acurácia dos resultados da segmentação.

O processo de segmentação é essencialmente empírico e, em geral, ajustável aos diferentes tipos de imagens, com limiares definidos face à complexidade dos alvos investigados. O nível que esta subdivisão deve ser realizada depende do problema a ser resolvido.

O presente trabalho objetivou testar diferentes valores para os critérios de similaridade e área, apontando os melhores resultados para o reconhecimento de segmentos referentes a plantios florestais. Ainda, faz parte do escopo deste trabalho realizar a avaliação do comportamento da forma dos segmentos gerados em função da variação dos limiares aplicados.

Este estudo se justifica por permitir a obtenção de resultados importantes que poderão preenchem uma lacuna de informação sobre o uso da técnica de segmentação na área florestal. 


\section{MATERIAIS E MÉTODOS}

A imagem utilizada neste trabalho é oriunda do Satélite SPOT 5, que apresenta resolução espacial de 10 metros nas bandas multiespectral e 5 metros na banda pancromática. A área de abrangência desta imagem é de 3.376 hectares e está localizada na cena 707-403, que recobre o município de General Carneiro, no Estado do Paraná (Figura 1).

Antecedendo o processamento, esta sub-cena passou pela operação de ortorretificação para um georreferenciamento adequado. Após o pré-processamento realizou-se a fusão das bandas pancromática e multiespectral, sobre a qual se efetuou a operação de segmentação. Portanto, a imagem processada possuía resolução espacial de $5 \mathrm{~m}$.

Foi utilizado o software Sistema de Processamento de Informações Georreferenciadas SPRING 4.2 (INPE), que apresenta o algoritmo segmentação por crescimento de regiões. Este algoritmo parte de um "pixel-semente" e, posteriormente, agrupa os pixels adjacentes que possuem características similares e que satisfazem os critérios de similaridade e de área. O primeiro critério é o limiar, abaixo do qual duas regiões são consideradas similares e então agrupadas. O segundo é o valor da área mínima dado em número de pixels, para que uma região seja individualizada.

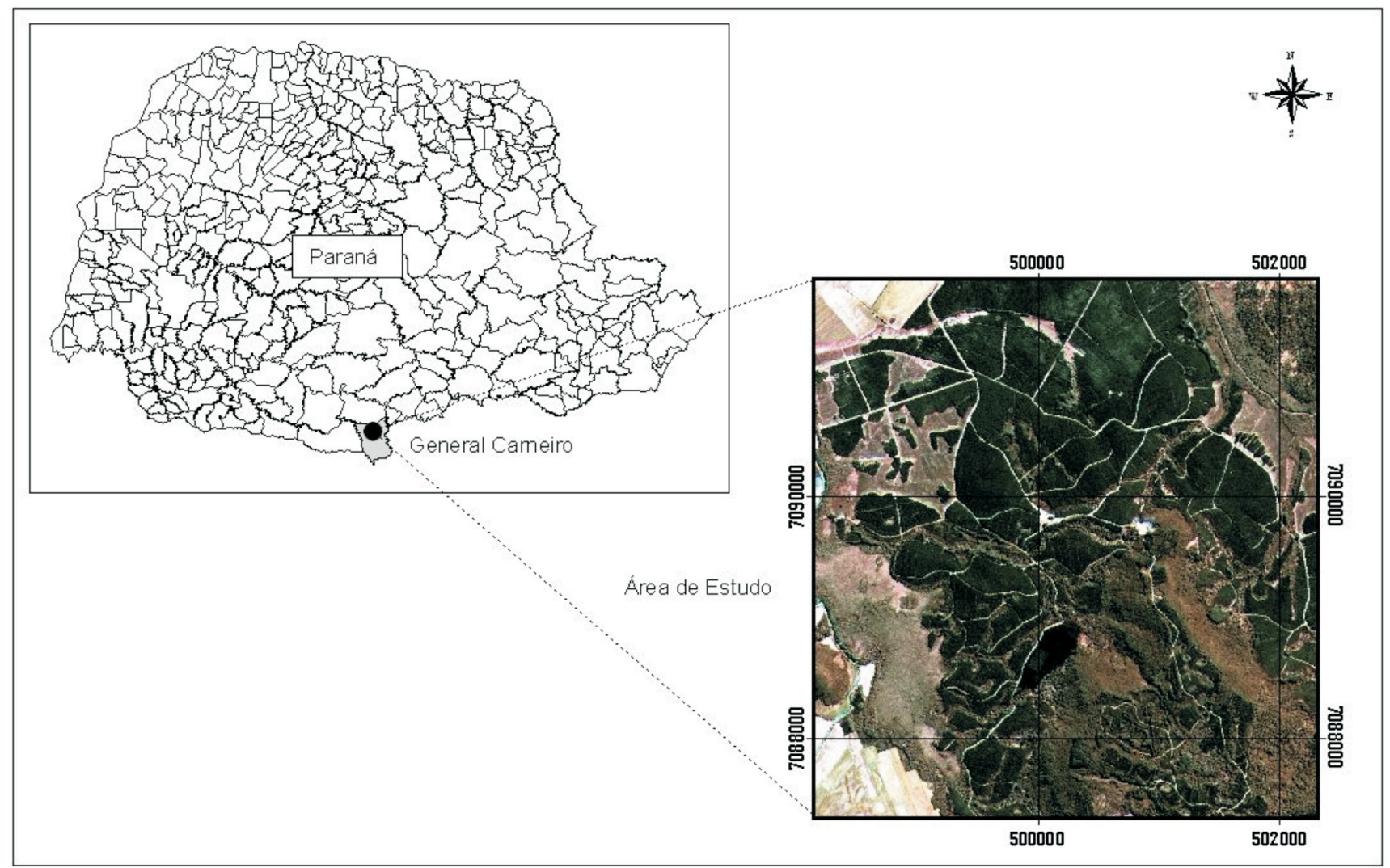

FIGURA 1 -Localização e imagem SPOT 5 da área de estudo

Figure 1 - Location of the studied area in Spot 5 image

Os limiares de similaridade e área foram definidos de forma sistemática. Para similaridade iniciou-se com valor 5 e efetuou-se um incremento de 5 níveis digitais até atingir o valor 15, passando, a partir deste valor, a efetuar um incremento de 10 níveis digitais. Para o limiar de área partiu-se do valor 100 , realizando um incremento de 100 pixels para os testes subsequentes. Foram testadas 24 combinações de limiares conforme apresentado na Figura 2. 


\section{Similaridade}

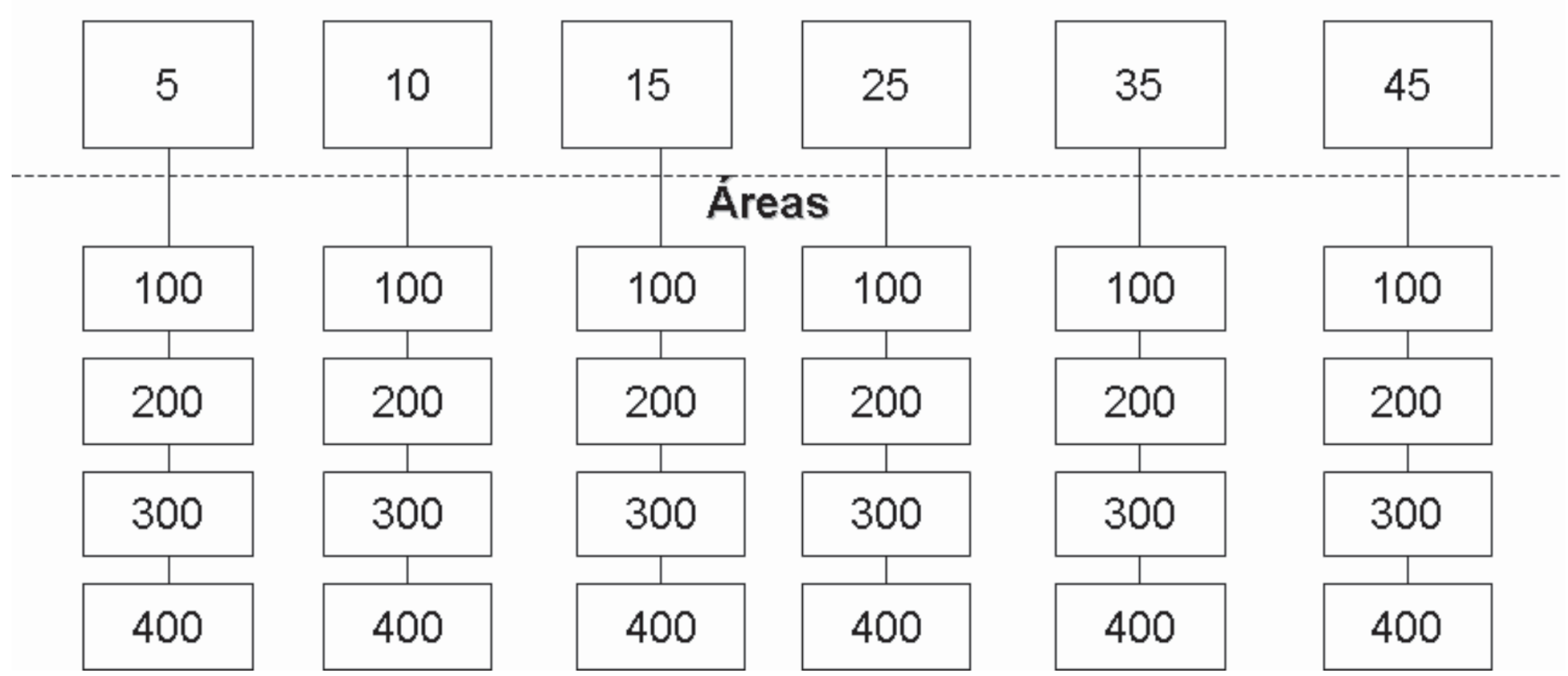

FIGURA 2 -Combinações dos pares de limiares testados neste trabalho Figure 2 - Thresholds pairs combinations tested in this work

Shoenmakers, Wilkinson e Schuten (1991) explicam que se os limiares forem muito baixos, o processo não atribuirá muitos pixels às classes. Por outro lado, se forem muito altos, os pixels representativos de diferentes classes serão incorretamente agrupados. Por esta razão, a imagem de bordas precisa ser avaliada para confirmar ou rejeitar os limiares adotados.

Os resultados gerados pela segmentação do SPRING apresentam-se na forma vetorial, mas ficam vinculados ao plano de informação referente à imagem. Para tornar possível o cálculo dos parâmetros para a análise da forma dos segmentos foi necessário transferir o resultado de cada par de limiares testado para um plano de informação de categoria temática.

A partir dos vetores gerados, foram realizados alguns cálculos visando avaliar a forma dos segmentos em cada um dos limiares testados. Estes cálculos foram: tamanho dos segmentos, perímetro dos segmentos e coeficiente de compacidade (CC). Estes cálculos foram gerados pelo software Arc View ( versão 3.1 (ESRI).

O parâmetro área média dos segmentos traz a idéia de quão segmentada a imagem resultante ficou e, da mesma forma, quanto mais segmentada a imagem, menor será o perímetro médio dos segmentos.

Ainda, realizou-se uma análise em relação ao coeficiente de compacidade que é obtido em função da área e do perímetro dos segmentos conforme equação 1.

$$
C C=\frac{P^{2}}{4 \Pi A} \quad \text { Equação } 1
$$

Onde:

$\mathrm{CC}=$ Coeficiente de compacidade;

$\mathrm{P}=$ Perímetro;

$\mathrm{A}=$ Área. 
Zhang (1996) apresenta dois métodos de análise de resultados da segmentação. O primeiro definido como método analítico - permite examinar e avaliar diretamente os algoritmos, não dependendo de experimentos e podendo ser aplicado sem que o algoritmo esteja completamente implementado. O segundo é o método empírico, que permite examinar e avaliar um algoritmo de forma indireta, por meio da aplicação de imagens-teste e medição da qualidade do resultado da segmentação. Lucca (1998) complementa que os métodos empíricos de discrepância permitem examinar e avaliar o desempenho de um algoritmo por meio da comparação da segmentação de uma imagem produzida pelo mesmo com uma segmentação de referência, sendo que ambas as segmentações são obtidas a partir do mesmo dado de entrada. No caso em que o dado de entrada é uma imagem real, a segmentação de referência é geralmente obtida por meio de uma segmentação manual da imagem de entrada baseada na interpretação visual.

Neste trabalho foi aplicado o método empírico de discrepância, onde o desempenho da variável forma foi avaliado sob o ponto de vista da capacidade de escolha dos melhores pares de limiares. Foi necessário realizar uma interpretação visual dos polígonos de plantios florestais (segmentação de referência) visando dar suporte na avaliação da qualidade da segmentação com os diferentes pares de limiares testados.

Desta forma, os resultados da segmentação foram sobrepostos com a área interpretada visualmente objetivando comparar os resultados obtidos com a chamada segmentação de referência. Este processo deu suporte à escolha dos melhores pares de limiares para a segmentação baseado no critério de interpretação visual, buscando-se os pares de similaridades que gerem resultados mais semelhantes aos da interpretação visual sem considerar um pós-processamento.

\section{RESULTADOS E DISCUSSÃO}

No Gráfico 1 estão apresentadas as áreas médias dos segmentos obtidas para cada combinação dos limiares. Observou-se que, com a interpretação visual, a área média dos polígonos ficou próxima a 55.276,24 $\mathrm{m}^{2}$. Este foi o valor referência para a realização das análises. Verificou-se que até a combinação de similaridade 45 e área 200 os valores médios não ultrapassaram os valores médios de referência, porém, as combinações 45 de similaridade e áreas 300 e 400 apresentaram valores superiores à média de referência, o que indica que alguns segmentos foram unidos, fato este que não atende ao objetivo da segmentação.

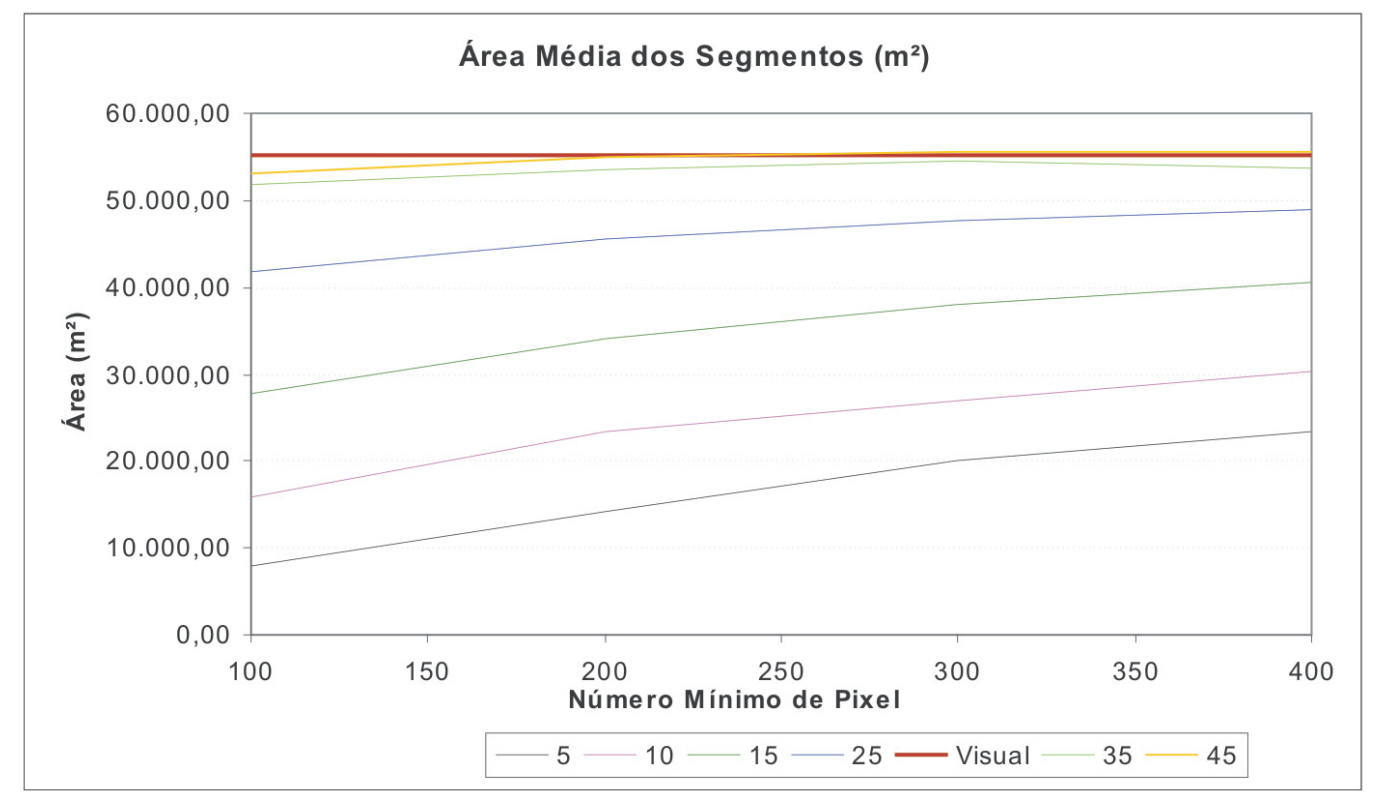

GRÁFICO 1 - Área média dos segmentos para os pares de limiares Graph 1 - Average area of the segments for the thresholds pairs 
Pode-se dizer que as similaridades de 25, 35 e 45 são as que apresentam melhores resultados em relação ao referencial. Também se observou que para estas similaridades, quanto maior o aumento da área mínima, melhor foi a detecção dos segmentos comparados ao referencial. O par que mais se aproximou da área média referencial foi o 45 de similaridade e 200 de área.

O perímetro apresentou um valor referencial de 1.066,71 metros. A medida que aumentamos o valor similaridade e área verificou-se uma tendência a aumentar o perímetro médio dos segmentos, pois as áreas são maiores (Gráfico 2). Porém, esta tendência não é linear. Os pares de limiares (similaridade e área) que mais se aproximaram do valor referencial de perímetro foram 10 e 200,15 e 100 , respectivamente.

Ainda, foi realizada uma análise em relação ao número médio de segmentos observados em cada um dos limiares. $\mathrm{Na}$ análise visual (referencial) foram gerados 183 polígonos. Notou-se que a similaridade de 35 para áreas de 300 e 400 e similaridade de 45 para as áreas 100, 200, 300 e 400 foram as que mais se aproximaram do referencial. Porém, cabe salientar que esta aproximação, para a similaridade de 35 e áreas de 200, 300 e 400 apresentam um número inferior de segmentos obtidos na interpretação visual. Este fato aponta para uma cautela na afirmação de que as similaridades de 25 e 35 são as melhores a serem adotadas, pois alguns segmentos foram dissolvidos em segmentos maiores não detectando as bordas originais.

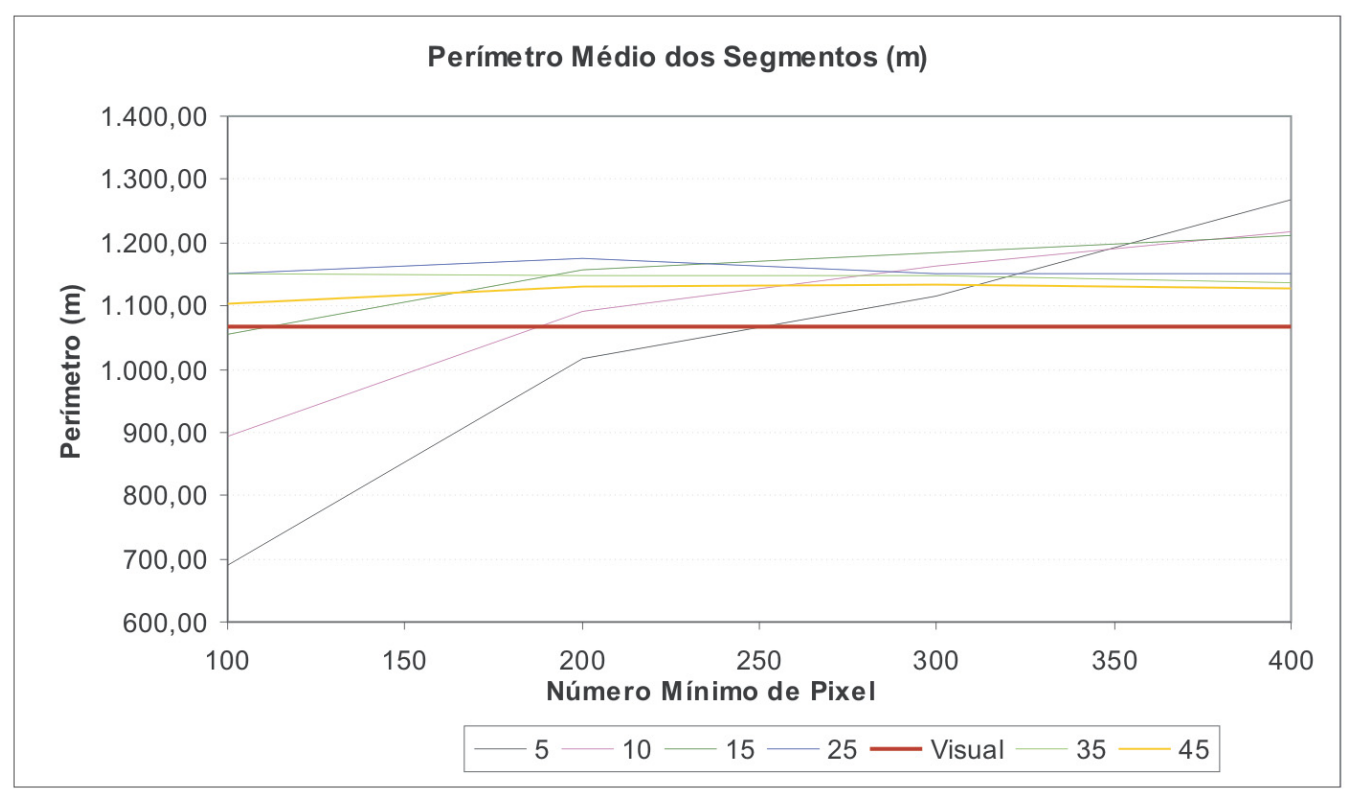

GRÁFICO 2 - Perímetro médio dos segmentos para os pares de limiares Graph 2 - Average length of the segments for the thresholds pairs

Diante de tais observações foi necessário lançar mão do índice que trata estas duas variáveis em conjunto para gerar, entre os limiares testados, um ranking de aproximação dos valores de compacidade referenciais. Observou-se que o valor referencial para este coeficiente de compacidade foi de 2,48. Observando a Tabela 1, percebe-se que a similaridade de 35 para áreas de 300 e 400 e similaridade de 45 para as áreas 100,200, 300 e 400 foram as que mais se aproximaram do referencial. 
TABELA 1 - Coeficientes de Compacidade para os limiares testados

Table 1 - Compactness index of the threshold limits tested

\begin{tabular}{ccccc}
\hline Similaridade & \multicolumn{4}{l}{ Área } \\
& $\mathbf{1 0 0}$ & $\mathbf{2 0 0}$ & $\mathbf{3 0 0}$ & $\mathbf{4 0 0}$ \\
\hline 5 & 5,37 & 6,60 & 5,70 & 6,43 \\
10 & 5,45 & 5,35 & 5,29 & 5,10 \\
15 & 4,92 & 4,77 & 4,42 & 4,26 \\
25 & 4,15 & 3,84 & 3,45 & 3,46 \\
35 & 3,22 & 3,05 & 2,95 & 2,96 \\
45 & 2,93 & 2,93 & 2,90 & 2,81 \\
\hline
\end{tabular}

Desta forma, o que se pode afirmar é que similaridades de 25, 35 e 45 apresentaram melhores resultados na identificação de polígonos de plantios florestais. Ainda, para estes índices, são indicadas áreas entre $200\left(5.000 \mathrm{~m}^{2}\right)$ e $300\left(7.500 \mathrm{~m}^{2}\right)$ pixels. Cabe ressaltar que estes valores são influenciados pela característica intrínseca do tamanho dos plantios florestais, que geralmente são áreas superiores a 1 hectare, tornando, assim, irrelevante a detecção de polígonos menores que este valor.

Desta forma, com base apenas nas variáveis avaliadas, fica um tanto quanto difícil afirmar qual par de limiar teve desempenho superior. Assim, optou-se por realizar uma escolha visual pelo método qualitativo. Portanto, visualmente o par de limiar que mais se aproximou da segmentação de referência foi 45 (similaridade) e 300 (área), conforme apresentado no Gráfico 3 e Figuras 3 e 4.

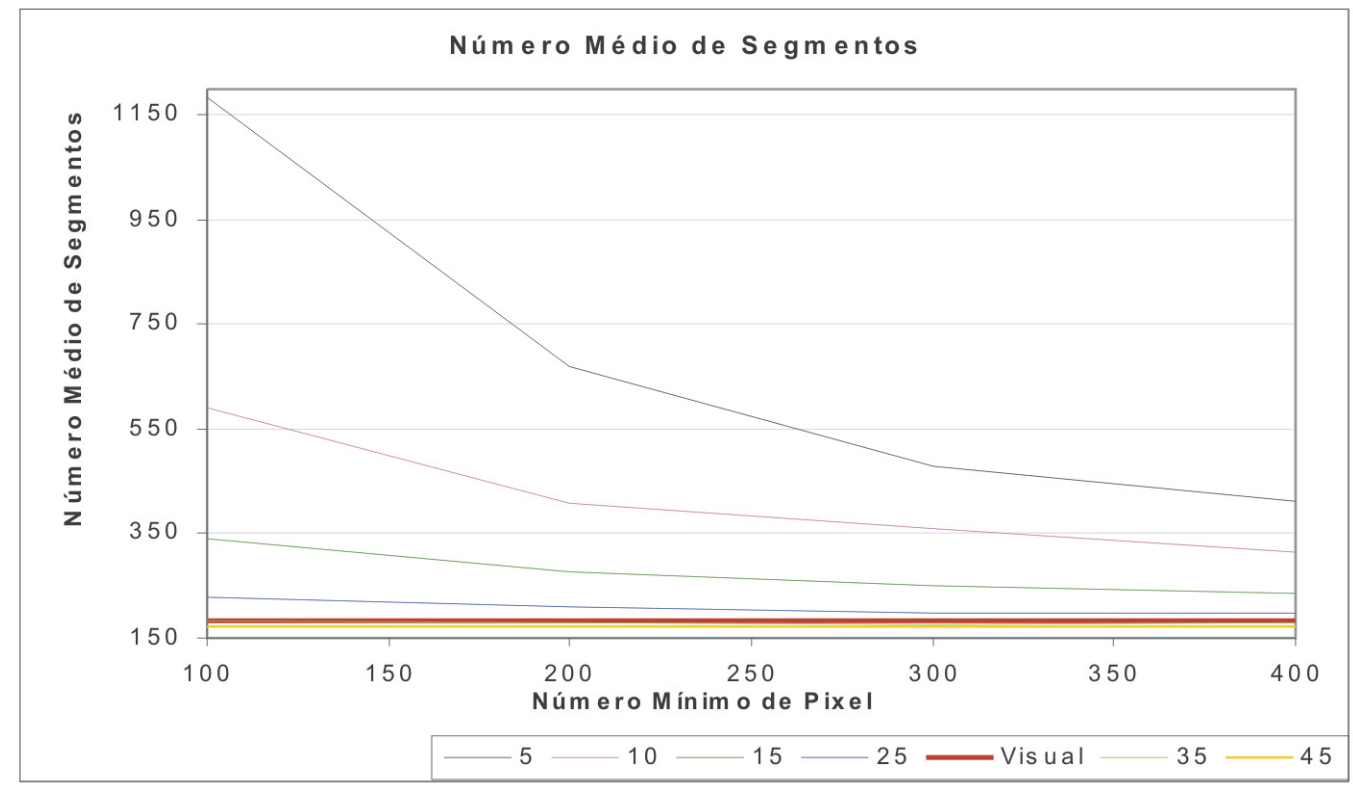

GRÁFICO 3 - Número médio dos segmentos para os pares de limiares Graph 3 - Average number of the segments for the thresholds pairs 


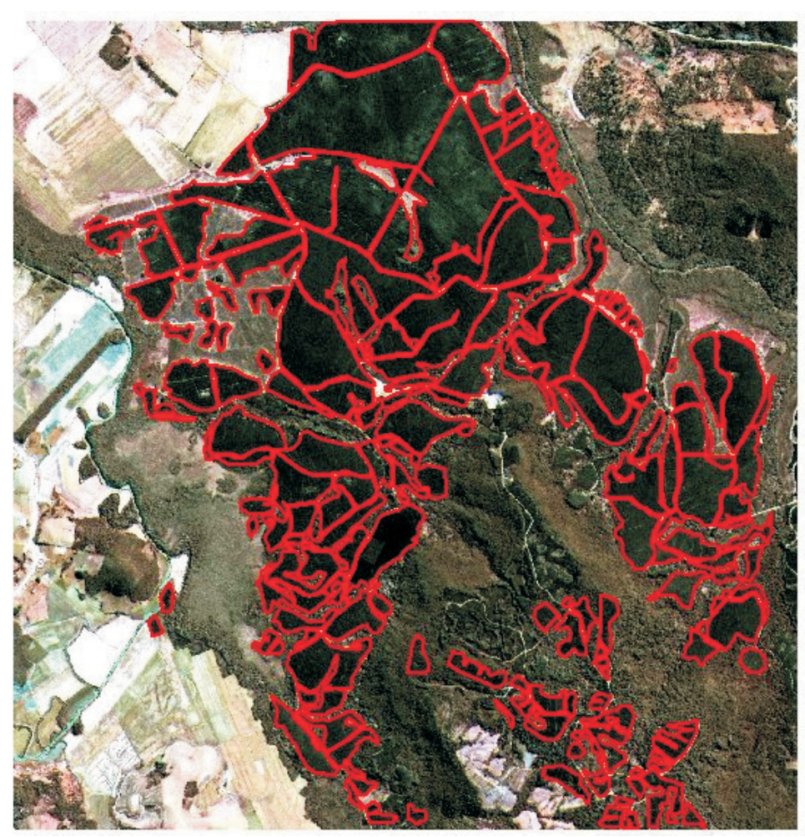

FIGURA 3 - Segmentação Visual (referência)

Figure 3 - Visual segmentation (reference)

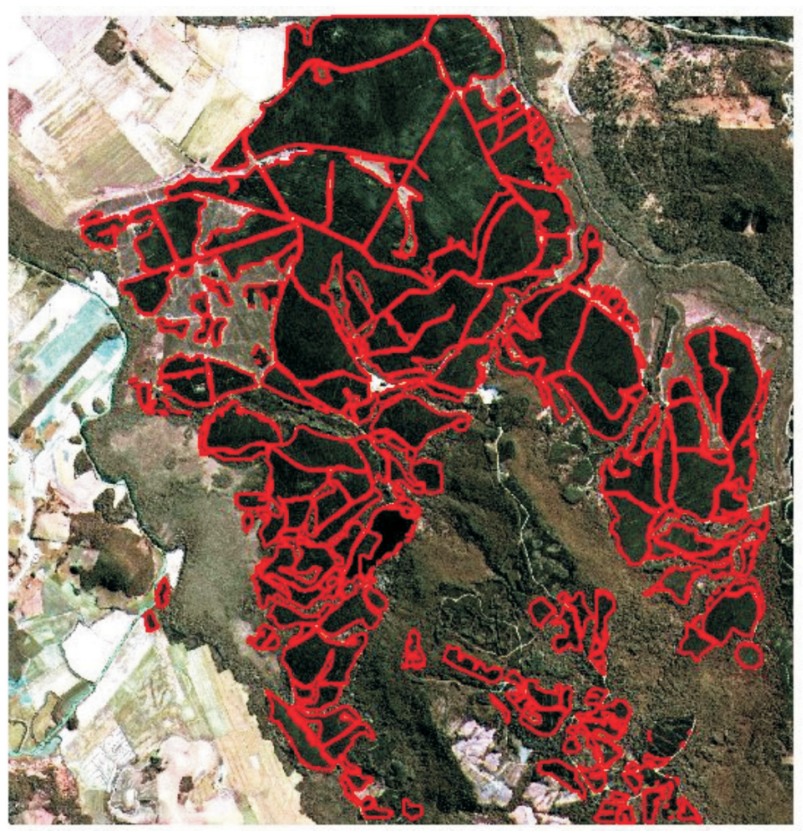

FIGURA 4 - Par de limiar 45 (similaridade) e 300 (área) Figure 4 - Threshold pair 45 (similarity) and 300 (area)

\section{CONCLUSÕES}

Pode-se concluir que as variáveis área, perímetro e coeficiente de compacidade trouxeram contribuições na avaliação da escolha dos pares de limiares, porém não conseguiram expressar claramente o melhor par. Cabe destacar ainda que, a partir do aumento dos valores de limiares, existe uma aproximação em relação à segmentação de referência, mas não de forma linear para todos os parâmetros;

Observou-se que o software SPRING 4.2 produziu resultados satisfatórios relativo à segmentação por crescimento de regiões, para imagem de alta resolução, com ênfase na detecção de plantios florestais;

Com base no critério visual, conclui-se que o par de limiar que mais se aproximou do referencial foi o 45 de similaridade com 300 de área. Conforme já discutido anteriormente, esses valores podem ter sido influenciados pelas características dos alvos analisados, ou seja, de possuírem tamanhos maiores que 1 hectare. Cabe ressaltar que é recomendável realizar estudos mais detalhados próximos a este par de valores, visando buscar possíveis combinações que possuam desempenho superior.

\section{REFERÊNCIAS}

COUTINHO, A. C. Segmentação e classificação de imagens Landsat-TM para o mapeamento dos usos da terra na região de Campinas, SP. 1997. 150 f. Dissertação (Mestrado em Ciências) - Curso de Pós-Graduação em Ciências, Departamento de Ecologia Geral do Instituto de Biociências, Universidade de São Paulo, São Paulo, 1997.

GONZALES, R. C.; WINTZ, P. Digital image processing. Boston: Prodding, Addison Wesley, 1987.

LUCCA, E. V. D. Avaliação e comparação de algoritmos de segmentação de imagens de radar de abertura sintética. 1998. 207 f. Dissertação (Mestrado em Sensoriamento Remoto) Instituto Nacional de Pesquisas Espaciais, São José dos Campos, 1998. 
MOREIRA, M. A. Fundamentos do sensoriamento remoto e metodologias de aplicação. São José dos Campos: INPE, 2001.

SCHOENMAKERS, R. P. H. M.; WILKINSON, G. G.; SCHOUTEN, T. E. Segmentation of remotely-sensed Images: a redefinition for operational applications. In: INTERNATIONAL GEOCIENCE REMOTE SENSING SYMPOSIUM, 91., 1991. Helsink. Proceedings... Helsink: IEEE, 1991. v. 2. p. 1087-1090.

VENTURIERI, A.; SANTOS, J. R. dos. Técnicas de classificação de imagens para análise de cobertura vegetal. In: ASSAD, E. D.; SANO, E. E. Sistema de informações geográficas. 2. ed. Brasília: Embrapa-SPI; Embrapa-CPAC, 1998. cap. 18. p. 351-371.

ZHANG, Y. J. A survey on evaluation methods for image segmentation. Pattern Recognition, v. 29, n. 8, p. 1335-1346, 1996.

Recebido: 23/04/2007

Received: 04/23/2007

Aprovado: $15 / 01 / 2008$

Approved: 01/15/2008 